\title{
FTIR and UV-vis study of chemically engineered biomaterial surfaces for protein immobilization
}

\author{
Herman Mansur ${ }^{\mathrm{a}, *}$, Rodrigo Oréfice ${ }^{\mathrm{a}}$, Marivalda Pereira ${ }^{\mathrm{a}}$, Zélia Lobato ${ }^{\mathrm{b}}$, \\ Wander Vasconcelos ${ }^{\mathrm{a}}$ and Lucas Machado ${ }^{\mathrm{c}}$ \\ ${ }^{a}$ Department of Metallurgy and Materials Engineering, Federal University of Minas Gerais, Brazil \\ ${ }^{\mathrm{b}}$ Department of Veterinary Medicine, Federal University of Minas Gerais, Brazil \\ ${ }^{\mathrm{c}}$ Department of Internal Medicine, School of Medicine, Federal University of Minas Gerais, Brazil
}

\begin{abstract}
The biomaterials research field has broadened in the last 3 decades, including replacement of diseased or damaged parts, assist in healing, correct and improve functional abnormality, drug delivery systems, immunological kits and biosensors. Proteins play crucial role in almost every biological system. They are involved in enzymatic catalysis, transport and storage, coordinated motion, mechanical support, immune protection, control of growth and cell differentiation among many others. The immobilization of proteins onto surface functionalized substrates has been one of the most promising areas in bioengineering field. It is important to note that the term immobilization can refer either to a temporary or to a permanent localization of the biomolecule on or within a support. Proteins have very particular chain configurations and conformations that promote high levels of specificity during chemical interactions. In the present work, we aimed to study the phenomenon of protein immobilization onto biomaterial with chemically engineered surface. We have tailored the surface of the porous gels of $\mathrm{SiO}_{2}$ with 5 different silane surface modifying agents: tetraethoxysilane (TEOS), 3-mercaptopropyltrimethoxysilane (MPTMS) and 3-aminopropyltriethoxysilane (APTES), 3-glycidoxypropyltrimethoxysilane (GPTMS) and 3-isocyanatopropyltriethoxysilane (ICPES). Fourier Transform Infrared Spectroscopy (FTIR) was used to characterize the presence of all specific chemical groups in the materials. The surface functionalized gels were then immersed in porcine insulin (PI) solutions for protein immobilization. The incorporation of protein within the gels was also monitored by FTIR spectroscopy. The kinetics of protein adsorption and desorption from the gel matrix in vitro tests were monitored by UV-visible spectroscopy. We could not observe any evidence of denaturation of insulin after its desorption from gel matrices using UV-visible spectroscopy technique. In vivo tests with adult male rats were used to verify the immobilized insulin bioactivity after implantation of different biomaterial with functionalized surfaces. Plasma glucose levels were obtained by using the Glucose GOD-ANA Colorimetric Assay. All surface modified materials have presented acute hypoglycemic peak response associated with the insulin bioactivity.
\end{abstract}

\section{Introduction}

The adsorption of protein onto surfaces is a topic of intense research. Proteins play crucial role in almost every biological system. They are responsible in one form or another for a variety of physiological functions. They are involved in enzymatic catalysis, transport and storage, coordinated motion, mechanical support, immune protection, generation and transmission of nerve impulse, control of growth and cell differentiation among many others. Protein adsorption on a biomaterial surface rules interactions between biomaterial and biological systems in a wide range. If the biomaterial is in contact to blood or any tissue, protein adsorption occurs almost immediately on the biomaterial surface and causes further

\footnotetext{
*Corresponding author. Rua Espírito Santo, 35/2 andar, 30160.030, Centro, Belo Horizonte, MG, Brazil. Tel.: +55 313238 1843; Fax: +55 313238 1815; E-mail: hmansur@demet.ufmg.br.
} 
activation of cellular and molecular cell components. Therefore, the desire to control, predict and manipulate protein adsorption at interfaces has been the driving force for the several hundred papers published in this area. According to the literature [1], a rigorous protein adsorption theory is yet to be developed. The multifaceted behavior of protein adsorption is related to their large size, complex architecture and mainly their physical-chemical stability. They have primary, secondary, tertiary and, in many cases, quaternary structure, with interactions ranging from weak to strong among chemical groups and side chains. As reported by several authors [2-7], surface chemistry can be used to manipulate protein-surface interaction by controlling the surface density and spatial distribution of chemical groups and ligands. A wide range of polar and non-polar silane which may be used to modify the surface of substrates such as glass, silica, alumina, polymers and metals are commercially available. The surface may be treated to decrease wettability or increase adhesion of the protein to substrate. The main challenge is based on engineering the surface in such a way that nonspecific reactions and interactions with the biological system must be inhibited and only very specific reactions will take place. It has an enormous potential for application as biomaterial implant, immunological kits, drug delivery systems and biosensors $[8,9]$.

Protein complex architecture has been a challenge for spectroscopists for many decades. The ability to describe and understand the conformation of proteins in solution or adsorbed onto surfaces pushed some characterization techniques to their limits. X-ray diffraction, NMR spectroscopy and many other methods have been used in protein studies [10]. Despite their importance, many methods have drawbacks related to the amount of protein material, difficulties on data analysis, quality of protein crystal and so forth. Fortunately, there are a few number of simple spectroscopy methods that have been developed over the years for assessing aspects of protein structure. UV-Vis and FTIR spectroscopy techniques are powerful methods to obtain relevant information for understanding protein adsorption to surfaces [10]. Protein quantitation and different interaction studies can be achieved by specific vibration bands associated with chemical groups and proper correlation curves in FTIR spectroscopy. The two dominant bands in any protein's absorbance FTIR-spectrum are the amide I and amide II absorptions. Light in the ultraviolet and visible regions (UV-Vis) promotes transitions between electronic states of molecules. In protein and protein components we focus on $\pi$-type molecular orbital. UV absorption spectroscopy in the range 250 $350 \mathrm{~nm}$ is based on $\pi-\pi^{*}$ electronic transition of some aromatic amino acids generally found in proteins. Such aromatic groups, phenyllalanine, tyrosine, and tryptophan are used as UV-Vis spectroscopic probes on protein studies. In addition to that, UV-Vis spectroscopy method has a broad range of utilization such as immunodiagnosis (ELISA), biochemical enzyme reactions (GOD-glucose oxidase), biosensors, just to name a few.

Porcine Insulin (PI) was chosen to be studied in the present work of protein-substrate interaction because of it is an extremely important peptide hormone. As mentioned by some authors [11], insulin is the physiologically dominant glucose regulatory factor. Insulin plays a vital role in living organisms with depressing the hepatic glucose production by inhibiting glycogenolysis and gluconeogenesis, stimulates peripheral glucose utilization, and therefore, decreases the plasma concentration of glucose. Therefore, the bioactivity response of the immobilized insulin in substrates would be detectable by glucose concentration analysis. Also, in the present study, porcine insulin molecule, that is made up by two chains (chain A and B), was used as a model system for further developmental investigations of the interfacial behavior of the larger proteins. 


\section{Experimental procedure}

Tetraethoxysilane $\mathrm{Si}\left(\mathrm{OC}_{2} \mathrm{H}_{5}\right)_{4}$ (TEOS > 98\%), 3-aminopropyltriethoxysilane (APTES), 3-mercaptopropyltrimethoxysilane (MPTMS) was supplied by Sigma-Aldrich. 3-Glycidoxypropyltrimethoxysilane (GPTMS) and 3-(triethoxysilyl)propylisocianate (TESPI) were supplied by Merk. Porcine insulin $\left(\mathrm{PI}>99.5 \%, \mathrm{M}_{\mathrm{r}}=5,778\right)$ was provided by Biobras SA. PBS solution (phosphate buffered solution) was prepared using the reagents: $\mathrm{Na}_{2} \mathrm{HPO}_{4}(>99.0 \%), \mathrm{NaH}_{2} \mathrm{PO}_{4}(>99.0 \%), \mathrm{Na}_{2} \mathrm{CO}_{3}(>99.5 \%)$ and $\mathrm{NaCl}(>99.0 \%)$.

\subsection{Porous network processing}

Porous glass matrix was obtained using TEOS and PBS solution with $\mathrm{pH}=7.40 \pm 0.05$. The gel surface was chemically patterned by adding the silane reagents 3-mercaptopropyltrimethoxysi lane (MPTMS), 3-aminopropyltriethoxysilane (APTES), 3-glycidoxypropyltrimethoxysilane (GPTMS) and 3-(triethoxysilyl)propylisocianate (TESPI) during the process of porous glass network formation (silane reagents shown in Table 1). These functional groups were chosen to address some important aspects of protein immobilization, such as hydrogen-bonds, Van der Waals and ionic forces, hydrophobic and hydrophilic interactions, sulfide bonds and steric hindrance. The sols were casted into 96-well-plate polystyrene molds, where gelation occurred. After thermal treatment of aging and drying, the gels of silica were immersed in PBS solution containing PI, to reach a final protein concentration of 1.0 weight\% in $\mathrm{SiO}_{2}$. The impregnated gels were subsequently dried for $48 \mathrm{~h} / 40^{\circ} \mathrm{C}$. These glass discs were produced with an average weight of $12 \pm 2 \mathrm{mg}$. All glass discs were accurately weighted before adsorption and desorption experiments, needed for mass balance calculations to quantify the concentration of PI immobilized into the porous glass matrices. The surface area of the porous network was characterized by $\mathrm{N}_{2}$ adsorption using B.E.T. method as previously reported in our work [12].

\subsection{FTIR spectroscopy}

Fourier Transform Infrared Spectroscopy (FTIR) was used to characterize the presence of specific chemical groups in the materials (Perkin-Elmer, Paragon 1000). Gels were milled and mixed with dried

Table 1

Silane reagents used as surface modifying chemical groups

\begin{tabular}{|c|c|c|}
\hline Formula & Surface modifying agents & Functional group (R) \\
\hline${ }_{\mathrm{EtO}}^{\mathrm{EtO}}$ OEt & $\begin{array}{l}\text { Tetraethoxysilane } \\
\text { (1) }\end{array}$ & $-\mathrm{OH}$ \\
\hline $\begin{array}{l}\mathrm{HSCH}_{2} \mathrm{CH}_{2} \mathrm{CH}_{2} \mathrm{Si}(\mathrm{OM})_{3} \\
\mathrm{H}_{2} \mathrm{~N}\left(\mathrm{CH}_{2}\right)_{3} \mathrm{Si}(\mathrm{OEt})_{3}\end{array}$ & $\begin{array}{l}\text { 3-Mercaptopropyl- trimethoxysilane } \\
\text { (2) }\end{array}$ & $-\left(\mathrm{CH}_{2}\right)_{3}-\mathrm{SH}$ \\
\hline$(\mathrm{MeO})_{3}-\mathrm{Si}-$ & $\begin{array}{l}\text { 3-Aminopropyl-triethoxysilane } \\
\text { (3) }\end{array}$ & $-\left(\mathrm{CH}_{2}\right)_{3}-\mathrm{NH}_{2}$ \\
\hline \multirow[t]{2}{*}{$\mathrm{OCN}\left(\mathrm{CH}_{2}\right)_{3}-\mathrm{Si}(\mathrm{OEt})_{3}$} & $\begin{array}{l}\text { 3-(Triethoxysilyl)-propylisocyanate } \\
\text { (4) }\end{array}$ & $-\left(\mathrm{CH}_{2}\right)_{3}-\mathrm{NCO}$ \\
\hline & $\begin{array}{l}\text { 3-Glycidoxy-propyl-trimethoxysilane } \\
\text { (5) }\end{array}$ & $-\left(\mathrm{CH}_{2}\right)_{3}-\mathrm{O}-\mathrm{Gly}$ \\
\hline
\end{tabular}


$\mathrm{KBr}$ powder. FTIR spectra were obtained within the range between $4000 \mathrm{~cm}^{-1}$ and $400 \mathrm{~cm}^{-1}$ during 64 scans. The incorporation of protein within the gels was also monitored by FTIR spectroscopy. We would like to point out that FTIR spectra were used as a qualitative reference of protein incorporation into the gel matrix. FTIR was also used to characterize the presence of specific chemical groups in the gel network, reflecting the effectiveness of the developed procedure for functionalization of porous glass.

\subsection{Protein release kinetics - "in vitro" assay}

This study employed a colorimetric method to determine protein concentrations, known as the Lowry assay [13]. The kinetics of protein (PI) desorption from the gel matrix was monitored by UV-visible spectroscopy (spectrophotometer Shimadzu UV-160A), with the concentration estimated through Lowry method readings at $660 \mathrm{~nm}$ wavelength [13]. The detailed protein analysis protocol has been previously reported in our work [14].

\subsection{Ultraviolet spectroscopic study of protein native conformation}

We have used solvent perturbation spectroscopy technique [10] (SPS) to verify denaturation effect of the developed process for incorporation of BSA and PI into the gels of silica and silica with functionalized surface. The SPS analysis was carried out through the UV spectra shift ("blue shift") obtained in the range of 250-300 nm when $4 \mathrm{M}$ guanidine hydrochloride ( $\mathrm{Gd}-\mathrm{HCl})$ was added to BSA and PI solutions. We have used the absorption differences between folded (native) and unfolded (denaturated) states of proteins. The effectiveness of the developed method for incorporation of proteins in porous gel glasses was evaluated by comparing pure gels of silica with gels with chemically functionalized surface.

\subsection{Bioactivity of insulin immobilized gels - "in vivo" assay}

Since the immobilization process may denature or otherwise inactivate the incorporated insulin, the bioactivity was investigated. Male rats were fasted overnight, anesthetized using ethyl ether reflux, weighted and $0.4 \mathrm{ml}$ blood samples were collected by intravenous catheter inserted in the jugular vein.

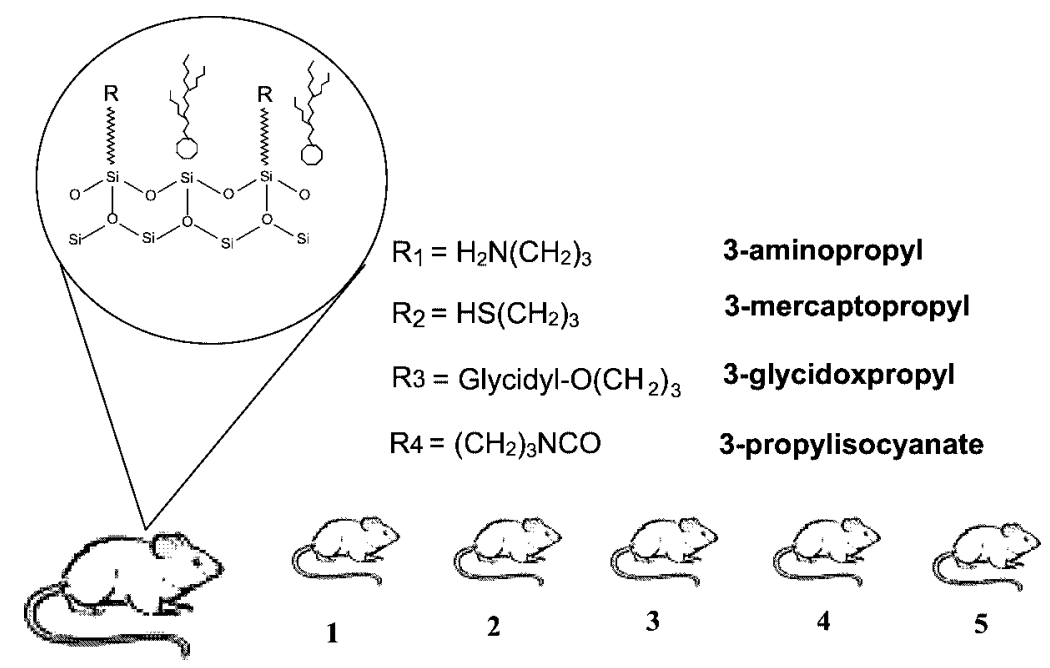

Fig. 1. Schematic representation of 5 different chemical groups of functionalized gel discs implanted in the rats. 
Subcutaneous implants with insulin incorporated of either pure silica gel discs or surface-modified gels were placed under the back skin of each rat. A schematic representation of the different gel implants in the rats is shown in Fig. 1. The rat was denied food and blood samples were collected at intervals of 20, 40, 60, 90, 120 and 150 min post-implanting. The heparinized blood samples were centrifuged at $7000 \mathrm{~g}$ for $10 \mathrm{~min}$, plasma was removed and saved for glucose monitoring. Plasma glucose levels were obtained by using the Glucose GOD-ANA Colorimetric Assay (Labtest Diagnostica S.A., Minas Gerais, Brazil), following the manufacturer's protocols with UV-Vis spectroscopy absorbance reading at $505 \mathrm{~nm}$.

\section{Results and discussion}

\subsection{Gel synthesis, surface functionalization and protein immobilization}

We have obtained the $\mathrm{SiO}_{2}$ porous glass and $\mathrm{SiO}_{2}$-functionalized glasses discs via chemical reaction of hydrolysis and policondensation of alkoxides. They were found to be optically transparent to visible light and mechanically stable to be handled. We have used FTIR spectroscopy for the characterization of the gel matrices synthesis and their surface functionalization. In Fig. 2, FTIR spectra of silica gels modified with 3-mercaptopropyltrimethoxysilane (MPTMS) are shown. The spectrum of pure silicagels (Fig. 2b) clearly reveals the peaks due to $\mathrm{Si}-\mathrm{O}-\mathrm{Si}$ bonds $\left(1080 \mathrm{~cm}^{-1}\right.$ and $\left.450 \mathrm{~cm}^{-1}\right)$ and $\mathrm{Si}-\mathrm{OH}$ bonds $\left(3500 \mathrm{~cm}^{-1}\right.$ and $\left.950 \mathrm{~cm}^{-1}\right)$. The spectrum in Fig. 2c is associated with the silica-gel modified with $1 \%$ molar with MPTMS. In order to enlarge the peaks due to MPTMS, a spectral subtraction involving the spectra of MPTMS gel and pure silica gel was performed and the result is exhibited in Fig. 2d. This spectrum (Fig. 2d) shows peaks at $2550 \mathrm{~cm}^{-1}$ that are characteristic of the MPTMS, as it can be observed in the spectrum of Fig. 2a. In Fig. 3, FTIR spectra of silica modified surface with 3-aminopropyltriethoxysilane (APTES) are shown. The reference spectrum obtained for pure silica-gel is shown in Fig. 3b, with the evidence of $\mathrm{Si}-\mathrm{O}-\mathrm{Si}$ bonds and $\mathrm{Si}-\mathrm{OH}$ bonds. The spectrum in Fig. 3c is from surface functionalized silica with 1 weight $\%$ of APTES. It can be noted the $-\mathrm{CH}$ stretching vibration bands (2850-3000 $\left.\mathrm{cm}^{-1}\right)$ due to the presence of propyl chain added with APTES. Therefore, FTIR data

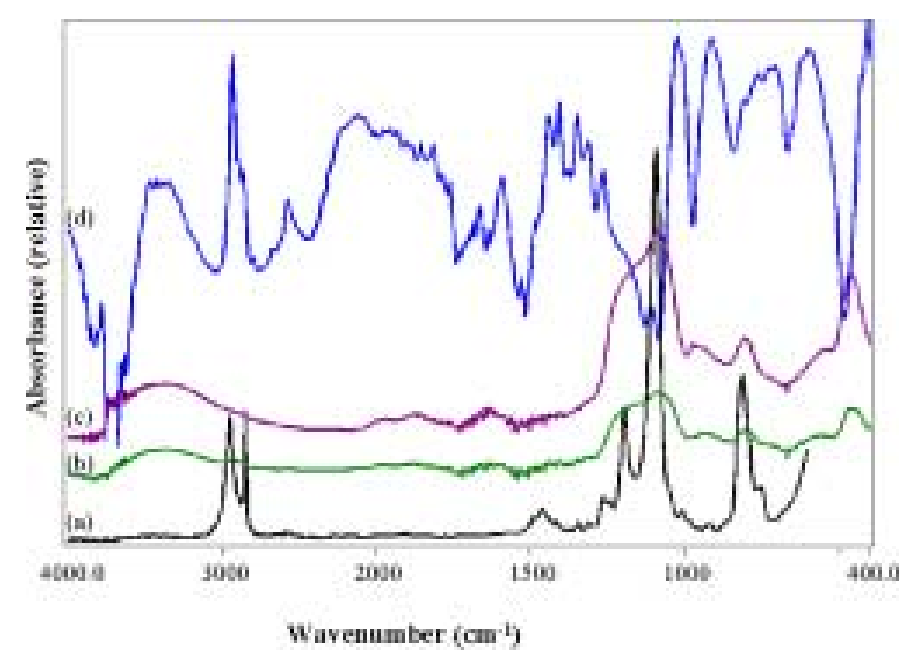

Fig. 2. FTIR spectra: (a) 3-mercaptopropyltrimethoxysilane (MPTMS); (b) pure silica-gel; (c) silica-gel modified with MPTMS and (d) spectral subtraction: spectrum (c)-spectrum (b). 


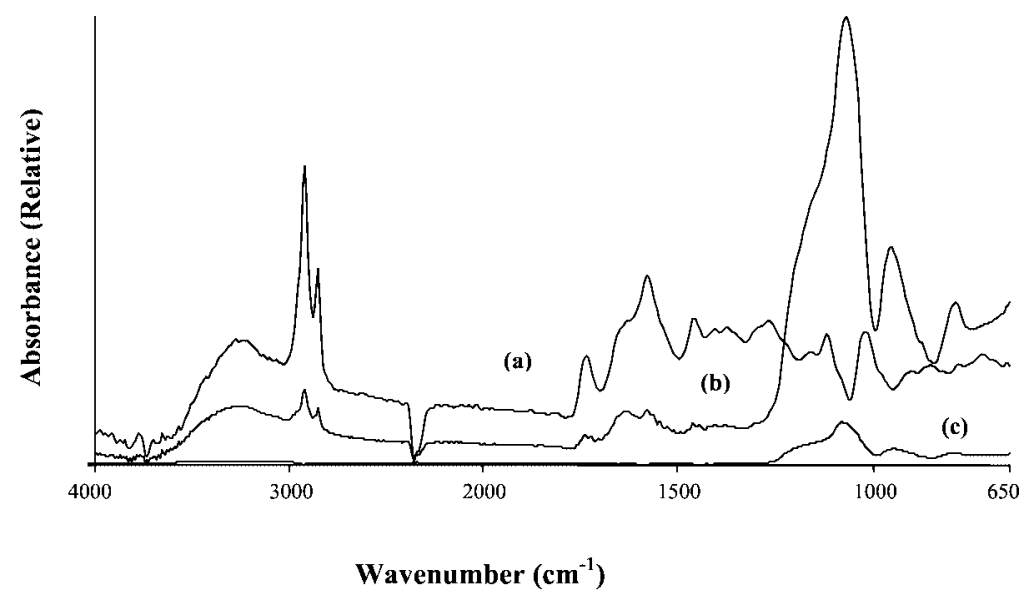

Fig. 3. FTIR spectra: (a) silica-gel modified with 3-aminopropyltriethoxysilane (APTES); (b) pure silica-gel; (c) 3-aminopropyltriethoxysilane (APTES).

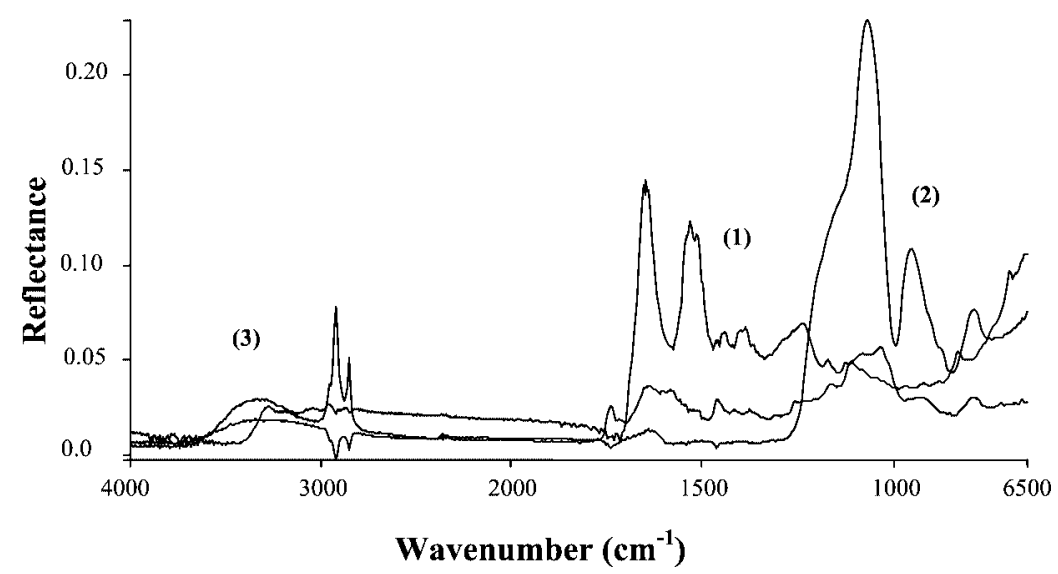

Fig. 4. FTIR spectra of porous glass and porous glass with PI incorporated. (1) reference spectrum of PI; (2) pure silica-gels; (3) the silica-gel modified with $1 \%$ MPTMS and $1 \%$ porcine insulin incorporated.

shown in Figs 2 and 3 gave strong evidence that the experimental procedure used in this work was successful in altering the chemistry of silica based gels. The presence of mercaptan $(-\mathrm{SH})$ and amine $(-\mathrm{NH})$ groups in the modified gels proves that the combination of different silane agents during synthesis of the gels can satisfactorily be used to produce materials containing specifically designed chemical functionalities. Different chemical species well dispersed within a silica matrix yield patterns of chemical activity that can be useful in tailoring the inorganic substrate towards interacting with a specific protein. Similar FTIR spectra were obtained for glass surface modifying groups, 3-glycidoxypropyltrimethoxysilane (GPTMS) and 3-(triethoxysilyl)propylisocianate (TESPI). Protein immobilization procedure was also monitored by FTIR spectroscopy method. Based on the literature [15], the peptide group, the structural repeat unit of proteins, gives up to 9 characteristic bands named amide (A, B, I, II, ..., VII). Amide I and amide II bands are two major bands of the protein infrared spectrum. The amide I band (between 1600 and $\left.1700 \mathrm{~cm}^{-1}\right)$ is mainly associated with the $\mathrm{C}=\mathrm{O}$ stretching vibration $(70-85 \%)$ and is directly related to the backbone conformation. Amide II results from the N-H bending vibration (40-60\%) and from the 
C-N stretching vibration (18-40\%). In Fig. 4, FTIR spectra of silica gels modified with mercaptopropyltriethoxysilane (MPTMS) are shown. Again, the spectrum of pure silica-gels (Fig. 4-2) reveals the peaks due to silicon bonds in the glass network. The spectrum in Fig. 4-3 is associated with the silica-gel modified with $1 \%$ molar with MPTMS and $1 \%$ porcine insulin (PI) incorporated. This spectrum (Fig. 4-3) shows peaks at $2550 \mathrm{~cm}^{-1}$ that are characteristic of the thiol $(-\mathrm{SH})$ and the presence of $-\mathrm{CH}$ stretching vibration bands (2850-3000 $\mathrm{cm}^{-1}$ ) mainly associated with the propyl group introduced by the functionalization of the gel surface with MPTMS. Figure 4-1 showed strong peaks at $\nu=1620-1680 \mathrm{~cm}^{-1}$ and $\nu=1480-1580 \mathrm{~cm}^{-1}$, mainly associated with amide-I and amide-II stretching vibration bands of porcine insulin (PI), respectively [16,17]. These results have clearly indicated the presence of proteins incorporated in the porous gel matrix. Also an increase in the peak intensity with raising the immobilized protein concentration in the porous glass matrix was observed.

\subsection{Protein adsorption kinetics - "in vitro" assay}

PI concentration in solution and immobilized into porous glass network was estimated by the adsorption spectra obtained for the respective protein standard. Based on the UV-Vis absorbance change of wavelength at $275 \mathrm{~nm}$, we were able to calculate the mass balance and the extension of each protein adsorption process. The calculation was done using Beer-Lambert law where the absorbance is proportional to the concentration of the protein in the solution. One typical result for the kinetics of PI adsorption in three different porous glass substrates (pure glass, thiol-functionalized and amino-functionalized glasses) is showed in Fig. 5. As it could be observed in Fig. 5, no major difference was detected on the insulin adsorption behavior of thiol and amino-modified silica gels compared to the pure silica glass. They all have shown a "flat off" pattern on insulin adsorption curve after approximately 30 minutes. Despite of that, it can be noted less insulin immobilized on both amino-terminated and thiol-terminated gels (15-18\%) when compared to the pure silica gel (approx. 25\%). We may assume some limitation on the protein immobilization onto the substrate associated with the steric hindrance of propyl groups present in APTES and MTMPS. Therefore, we can conclude that the silane surface modifying agents may the used on protein adsorption control kinetics.

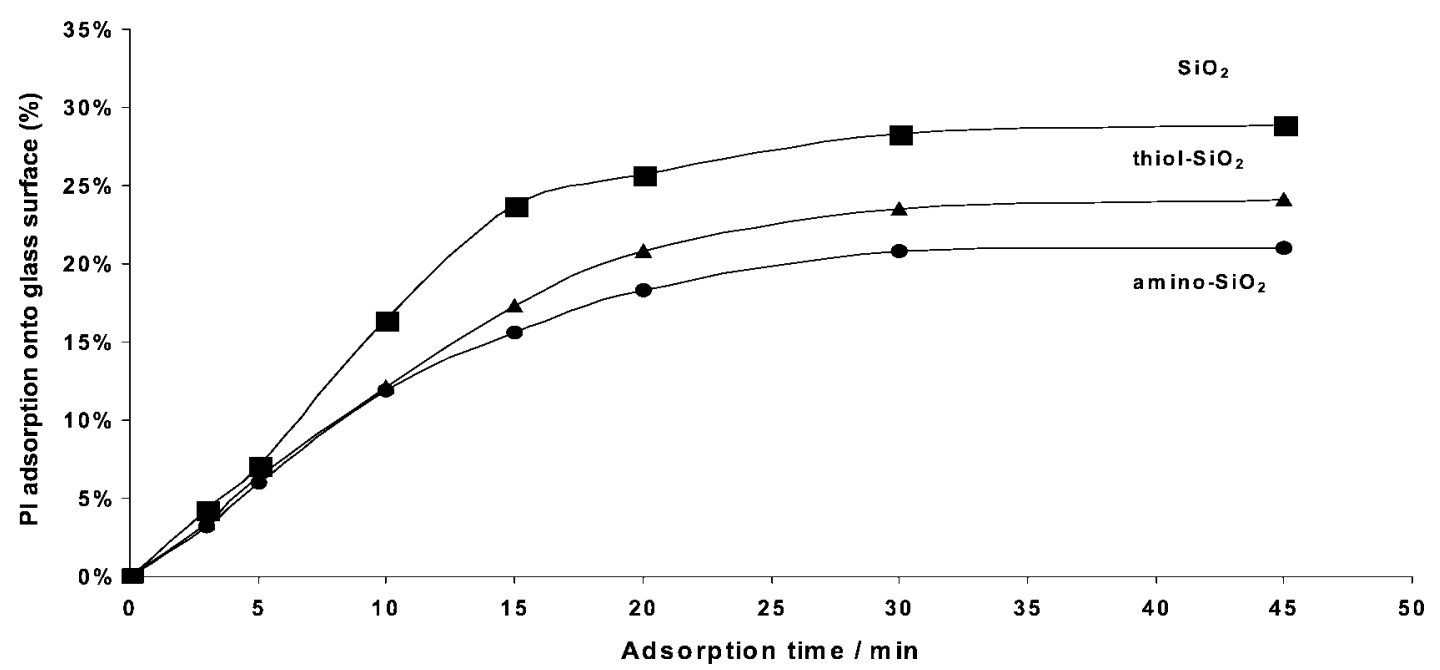

Fig. 5. Calculation of PI adsorption in three different porous glass substrates obtained by UV-Vis spectroscopy absorbance at wavelength of $275 \mathrm{~nm} ;(\mathbf{\square}) \mathrm{SiO}_{2} ;(\boldsymbol{\Delta}) \mathrm{R}-\mathrm{SH}$ terminated glass; $(\bullet) \mathrm{R}-\mathrm{NH}_{2}$ terminated glass. 


\subsection{Ultraviolet spectroscopic study of protein native conformation stability}

We have used solvent perturbation spectroscopy [10] (SPS) to evaluate if the incorporation process of $\mathrm{PI}$ into the gels of $\mathrm{SiO}_{2}$ and $\mathrm{SiO}_{2}$ with chemically functionalized surface has denaturated the incorporated proteins. We have used the absorption differences between the folded (native) and unfolded (denaturated) states of proteins to investigate their stability. After adding $4 \mathrm{M}$ guanidine hydrochloride to PI solutions, released from the porous $\mathrm{SiO}_{2}$ matrices, the UV spectra (not shown) have indicated a "blue shift" on the maximum of absorbance peaks. These results have given strong evidence that no detectable denaturation of PI has occurred in the immobilization procedure developed in this work. This observation is of major importance considering biological activity of proteins in living organisms. The denaturation process of proteins is mainly related to modification of the three-dimensional conformation structure. These SPS findings were confirmed through "in vivo" evaluation experiments.

\subsection{Bioactivity of insulin immobilized gels implants - "in vivo"}

We have conducted in vivo assay using PI incorporated into gel network because of the high specificity and sensibility of this characterization procedure. Figure 6 showed a typical in vivo response of the glass implanted material. It can be clearly observed the bioactivity of the insulin incorporated in the silica glass disc by decreasing the plasma concentration of glucose compared to the control rat without PI incorporated (central strip). After 90 minutes the glucose level is less than $40 \mu \mathrm{g} / \mathrm{ml}$ indicating strong response to the PI implanted. It could be observed hyperglycemic peaks for all samples on the first 10 minutes post-implantation (Fig. 7). Such fact is generally caused by the experiment stress over the rats. After that, pure silica-gels and surface modified gel implants have presented a similar trend on blood sugar level, with an acute hypoglycemic effect observed in these implanted animals compared to glucose level of the control group. A significant reduction from about $120 \mu \mathrm{g} / \mathrm{ml}$ to less than $40 \mu \mathrm{g} / \mathrm{ml}$ within 30 minutes was verified due to strong insulin response [11]. In Fig. 7 is shown the PI post-implantation curves for five different functionalized substrates. The central band represents range of blood glucose level of the control rat with implant of pure $\mathrm{SiO}_{2}$ glass (without PI). In few cases we have even observed convulsive response in rats, mainly due to dramatically reduction of rat blood sugar level induced by

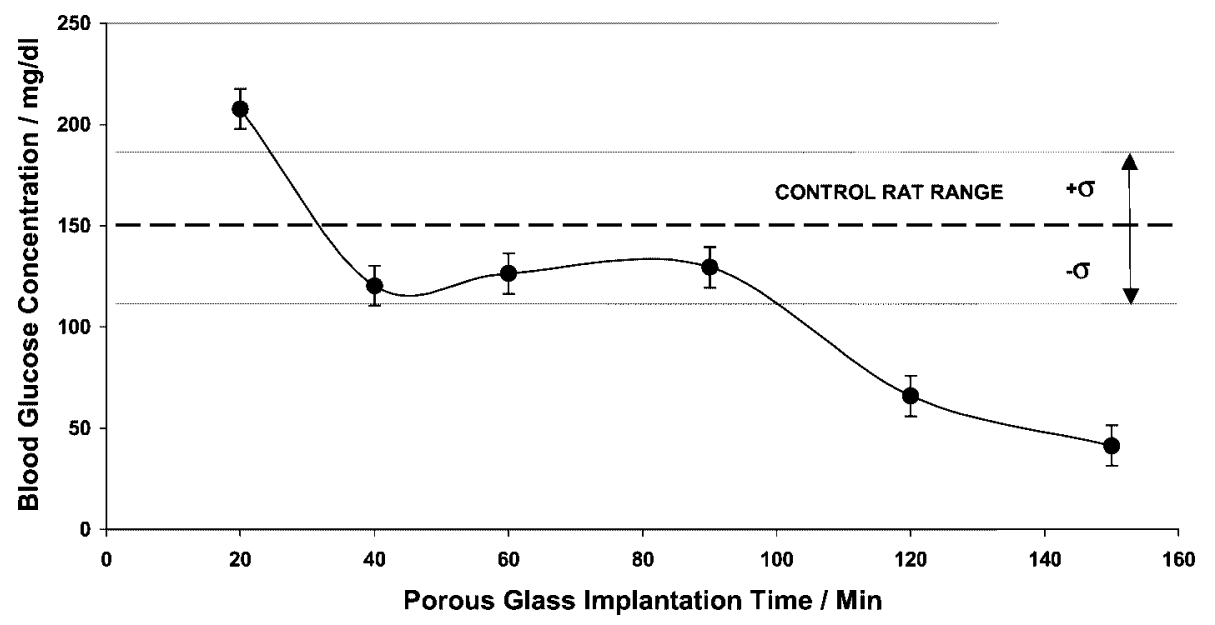

Fig. 6. Plasma glucose concentration in response to insulin administration time; (1) rat with implant of porous $\mathrm{SiO}_{2}$ containing $1 \%$ weight of PI; (2) control rat with implant of pure $\mathrm{SiO}_{2}$ glass (without PI). 


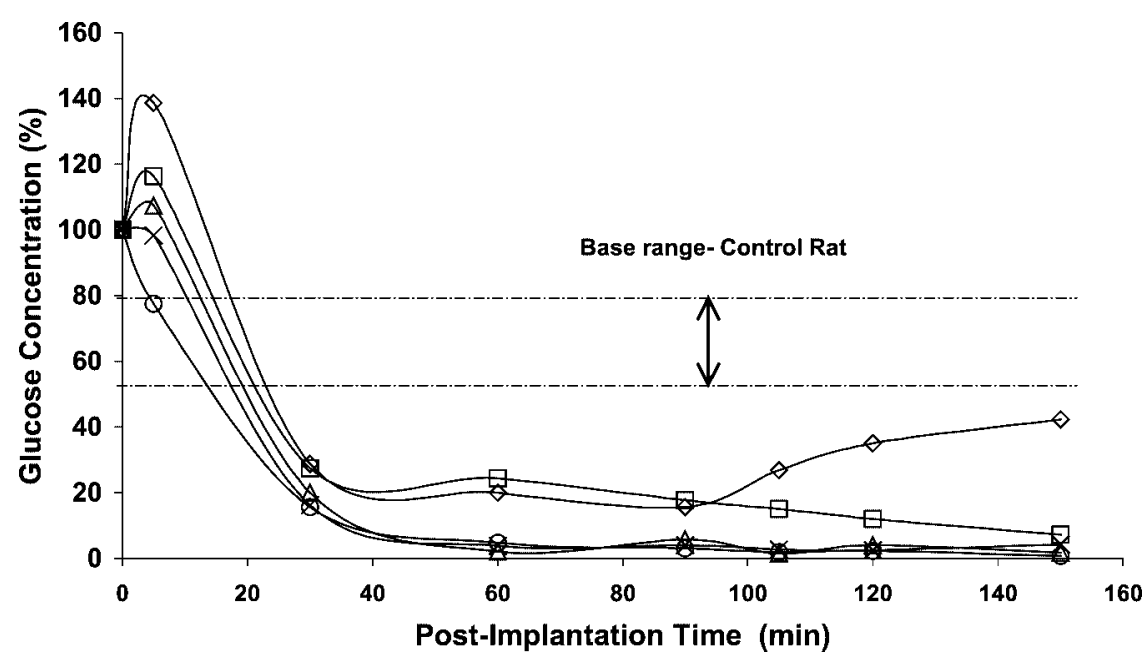

Fig. 7. Plasma glucose concentration in response to insulin administration time; 5 different silica-gel surface; central band represents range of blood glucose level of the control rat with implant of pure $\mathrm{SiO}_{2}$ glass (without PI).

PI administration through the subcutaneous gel implant. Therefore, we assume that the incorporation process of protein into $\mathrm{SiO}_{2}$ glass network has retained the bioactivity and has not caused any detectable loss of its bioactivity. Also, no major effect on the PI response was observed among the surface modified substrates when comparing the blood glucose concentration curves.

\section{Conclusion}

FTIR results have shown that the experimental procedure developed in this work was successful in altering the chemistry of silica based gels. The synthesis of the gels with surface modifying groups can satisfactorily be used to produce materials containing specifically designed chemical functionalities. Porcine insulin immobilization in the gel network was monitored by FTIR technique. Also, UV spectroscopy (SPS) results have given strong evidence that no detectable denaturation of PI has occurred in the immobilization procedure developed in this work. This observation is of major importance considering biological activity of proteins in living organisms. The SPS findings were confirmed through "in vivo" evaluation experiments, where an acute hypoglycemic effect observed in the implanted animals caused by the insulin bioactivity. Also, no major effect on the PI bioactivity difference was observed among the surface modified substrates when comparing the blood glucose concentration curves.

\section{References}

[1] V. Hlady and J. Buijs, Protein adsorption on solid surfaces, Curr. Opin. Biotechnol. 7 (1996), $72-77$.

[2] I. Gill and A. Ballesteros, Encapsulation of biologicals within silicate, siloxane, and hybrid sol-gel polymer. An efficient and generic approach, Journal of The American Chemical Soc. 120 (1998), 8587-8598.

[3] B.C. Dave, B. Dunn, J.S. Valentine and J.I. Zink, Sol-gel encapsulation methods for biosensors, Analyt. Chem. 66 (1994), $1120-1127 \mathrm{~A}$

[4] L.M. Ellerby, C.R. Nishida, F. Nishida, S.A. Dunn, B.Yamanaka, J.S. Valentine and J.I. Zink, Encapsulation of proteins in transparent porous silicate glasses prepared by the sol-gel method, Science 255 (1992), 1113-1115.

[5] L.L. Hench and J.K. West, Chem. Rev. 90 (1990), 33. 
[6] J. Livage, J.Y. Barreau, J.M. Da Costa and I. Deportes, Optical detection of parasitic Protozoa in sol-gel matrices, SPIE, Sol-Gel Optics III 2288 (1994), 493-503.

[7] C. McDonagh, B.D. MacCraith and A.K. McEvoy, Tailoring of sol-gel films for optical sensing of oxygen in gas and aqueous phase, Anal. Chem. 70 (1998), 45-50.

[8] R. Zusman, D.A. Beckman, I. Zusma and R. Brent, Purification of sheep immunoglobulin-G using protein-A trapped in sol-gel glass, Analyt. Biochem. 201 (1992), 103.

[9] B.D. Ratner, A.S. Hoffman, F.J. Schoen and J.E. Lemons, in: Biomaterials Science: An Introduction to Materials in Medicine, Academic Press, New York, 1996.

[10] R. Copeland, in: Methods for Protein Analysis, Chapman Hall, New York, USA, 1994.

[11] N. Yamaguchi, Sympathoadrenal system in neuroendocrine control of glucose, Can J. Physiol. Pharmacol. 70 (1992), $167-205$.

[12] H.S. Mansur, Z.P. Lobato, R. Orefice, W.L. Vasconcelos and L.J.C. Machado, Surface functionalization of porous glass networks: Effects on BSA and PI immobilization, Biomacromolecules 1 (2000), 789-797.

[13] O.H. Lowry, N.J. Rosenbrough, A.L. Farr and R.J. Randall, Protein measurement with the Folin phenol reagent, J. Biol. Chem. 193 (1951), 265-275.

[14] H.S. Mansur, Z.P. Lobato, R.L. Orefice, W.L. Vasconcelos, L.J.C. Machado and E. Mansur, Adsorption/desorption behavior of BSA and PI on chemically patterned porous gel networks, Adsorption 7 (2001), 105-116.

[15] K.K. Chittur, FTIR/ATR for protein adsorption to biomaterial surfaces, Biomaterials 19 (1998), 357-369.

[16] C.F. Lu, A. Nadarajah and K.K. Chittur, A comprehensive model of multiprotein adsorption on surfaces, Journal of Colloid and Interface Science 168 (1994), 152-161.

[17] M. Malmsten, in: Biopolymers at Interfaces: Protein on Surfaces, K. Chittur, ed., Marcel Dekker Inc., New York, USA, 1998, p. 143. 


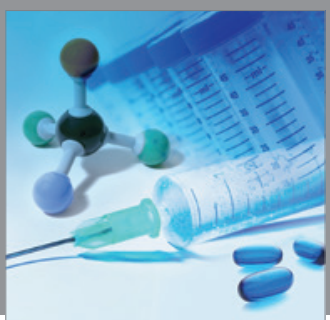

International Journal of

Medicinal Chemistry

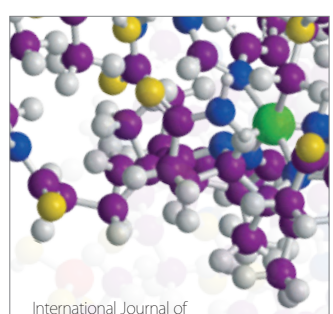

Carbohydrate Chemistry

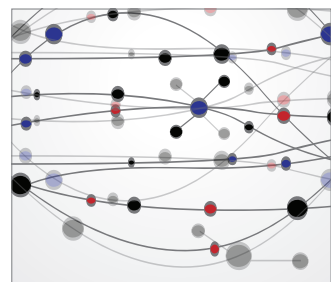

The Scientific World Journal
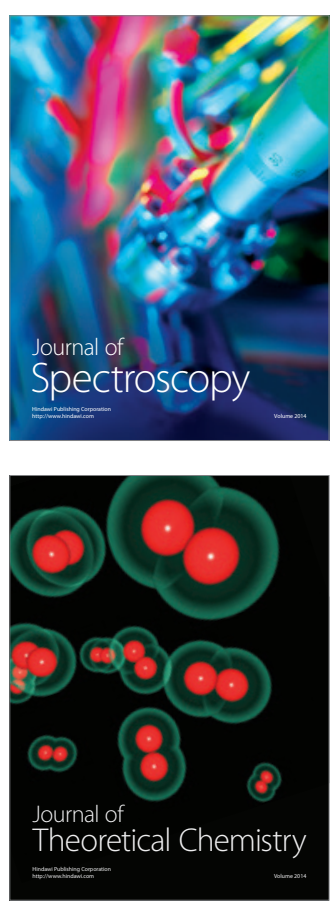
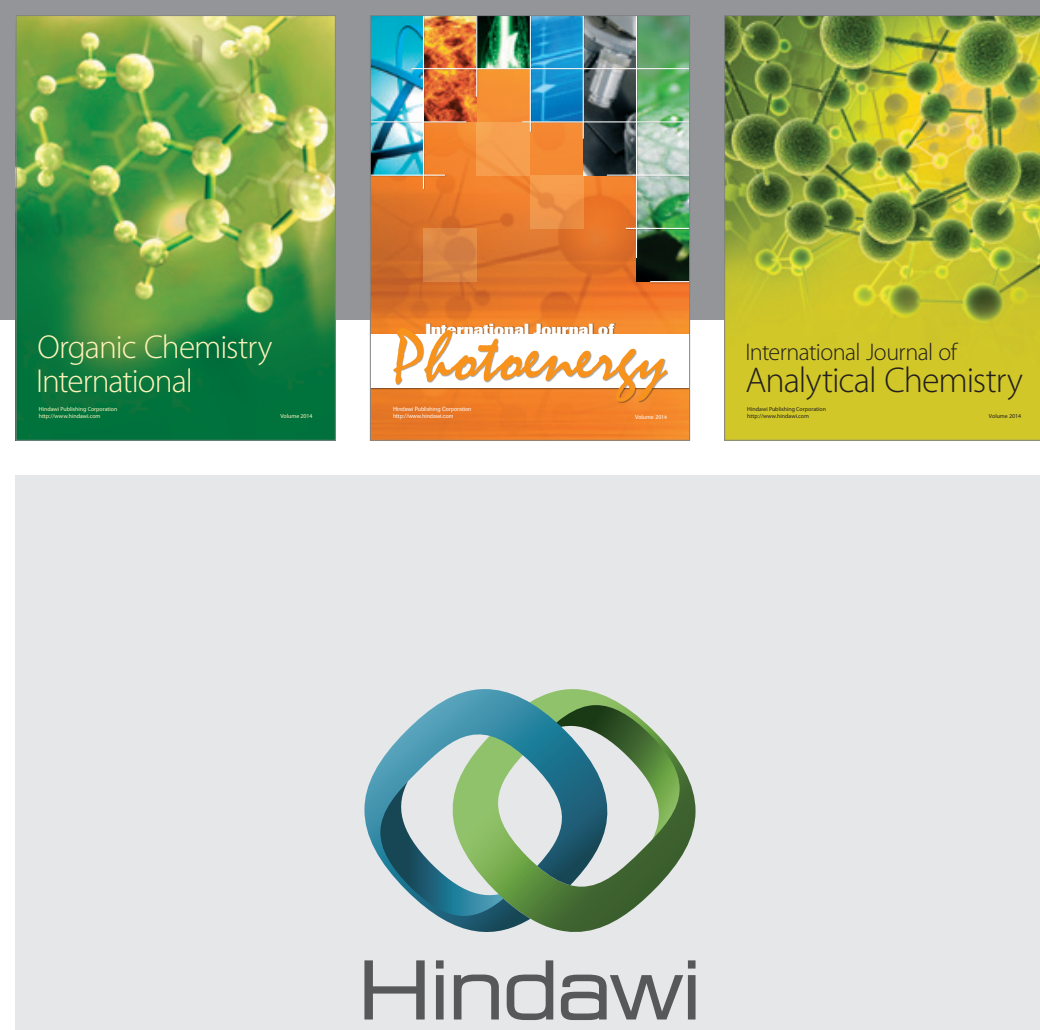

Submit your manuscripts at

http://www.hindawi.com
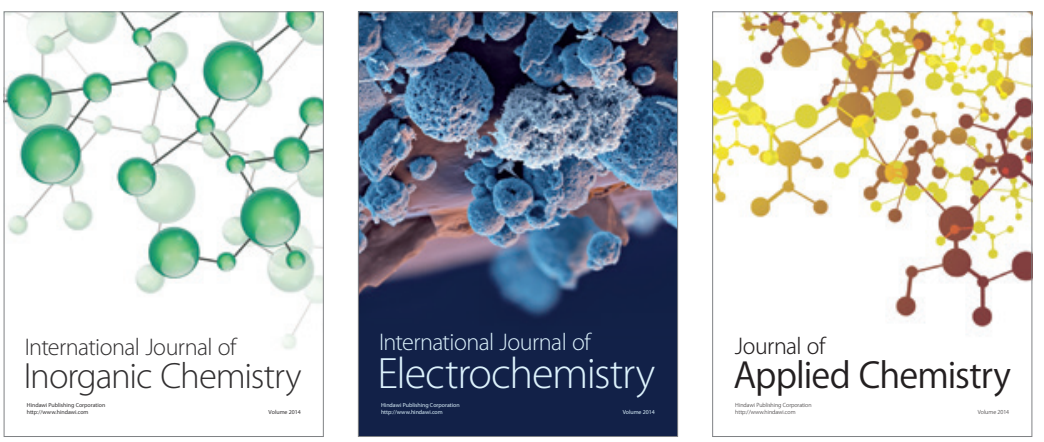

Journal of

Applied Chemistry
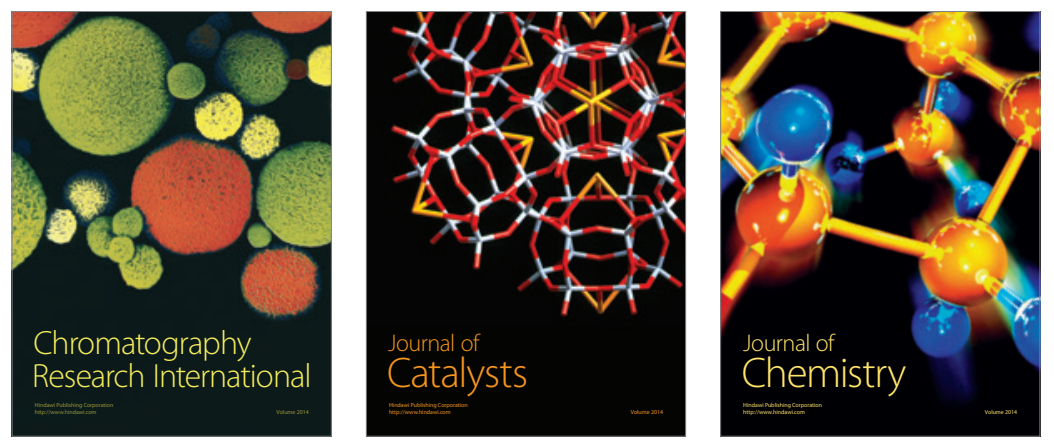
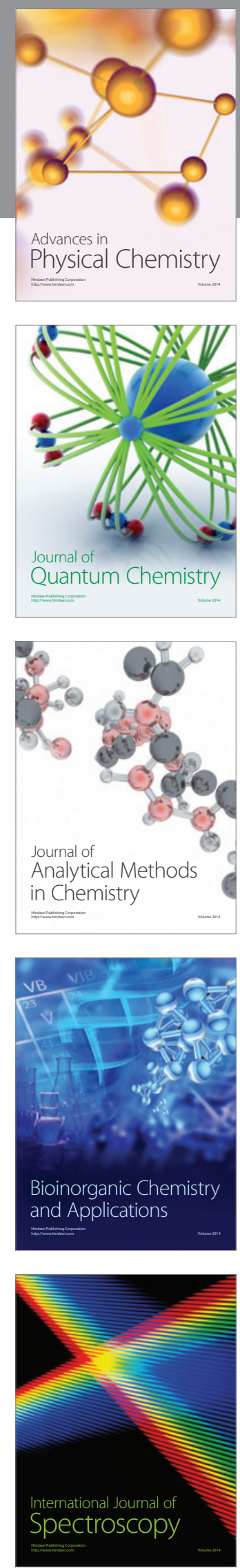